\title{
Satisfação e qualidade de vida de idosos institucionalizados usuários e não usuários de prótese total
}

\author{
Satisfaction and quality of life of institutionalized elderly users and non-users of complete dentures \\ Satisfacción y calidad de vida de los usuarios ancianos institucionalizados y no usuarios de prótesis \\ total
}

Recebido: 03/04/2021 | Revisado: 11/04/2021 | Aceito: 12/04/2021 | Publicado: 23/04/2021

Laís Samara Juliano de Carvalho ORCID: https://orcid.org/0000-0001-7680-3078 Universidade de Pernambuco, Brasil E-mail: laissamaraodonto@gmail.com

Nathália Larissa Bezerra Lima

ORCID: https://orcid.org/0000-0003-1934-2708 Universidade de Pernambuco, Brasil E-mail: nathalialbl.123@gmail.com

Jéssica Caroline Carvalho Souza

ORCID: https://orcid.org/0000-0003-0565-2449 Universidade Federal do Rio Grande do Norte, Brasil E-mail: jessodonto.14@gmail.com

Dayane Bezerra Rêgo

ORCID: https://orcid.org/0000-0002-0918-9458 Universidade de Pernambuco, Brasil E-mail: dayanebrego@gmail.com

Desirée de Paula Barroso Menezes

ORCID: https://orcid.org/0000-0001-7755-7294 Universidade Federal do Rio Grande do Norte, Brasil

E-mail: demenezesodonto@hotmail.com

Raniel Fernandes Peixoto

ORCID: https://orcid.org/0000-0002-6845-0767 Universidade Federal do Ceará, Brasil E-mail: raniel.peixoto@ufc.br

\begin{abstract}
Resumo
Objetivo: Comparar a satisfação e a qualidade de vida de idosos usuários e não usuários de prótese total (mas que necessitam de prótese). Métodos: Trata-se de um estudo transversal. A amostra foi constituída por 62 idosos, sendo 31 usuários (G1) e 31 não usuários (G2) de prótese total (PT). Para todos os participantes, questionários de satisfação (Gurgel et al., 2015) e qualidade de vida (Oral Health Impact Profile for edentulous - OHIP-Edent) foram aplicados. Os dados foram analisados por meio de análise descritiva, seguido por testes estatísticos para avaliar diferenças na satisfação e qualidade de vida entre usuários e não usuários de PT. Resultados: A análise comparativa dos aspectos sóciodemográficos entre G1 e G2 mostrou nenhuma diferença estatisticamente significante ( $\mathrm{p}>0,05)$. A maioria foi do gênero feminino $(n=51 ; 82,3 \%)$, casado $(n=20 ; 32,3 \%)$, aposentado $(n=45 ; 72,6 \%)$, renda de até 1 salário $(n=44$; $71,0 \%)$ e ensino fundamental incompleto $(n=35 ; 56,5 \%)$. A satisfação foi significativamente maior nos usuários de PT em todos os parâmetros avaliados $(\mathrm{p}<0,05)$. Dentre os indicadores de qualidade de vida, somente dor física $(\mathrm{p}=0,123)$ e limitação psicológica $(\mathrm{p}=0,087)$ não mostraram diferença estatisticamente significante $(\mathrm{p}>0,05)$. Conclusão: Dentro das limitações deste estudo, a reabilitação com PT promoveu um impacto positivo na vida dos idosos, ao garantir uma maior satisfação e melhor qualidade de vida. Adicionalmente, as escalas EVA e adjetiva apresentaram uma concordância substancial.
\end{abstract}

Palavras-chave: Prótese total; Satisfação do paciente; Qualidade de vida.

\section{Abstract}

Objective: To compare the satisfaction and quality of life of complete denture elderly users and non-users, (but who need denture). Methods: This is a cross-sectional study. The sample consisted of 62 elderly, 31 users (G1) and 31 nonusers (G2) of complete denture (CD). For all participants, satisfaction (Gurgel et al., 2015) and quality of life (Oral Health Impact Profile for edentulous - OHIP-Edent) questionnaires were applied. Data were analyzed by descriptive analysis, followed by statistical tests to evaluate differences in satisfaction and quality of life before CD users and non-users. Results: The comparative analysis of the socio-demographic aspects between G1 and G2 showed no statistically significant difference $(\mathrm{p}>0.05)$. The majority were female $(\mathrm{n}=51 ; 82.3 \%)$, married $(\mathrm{n}=20 ; 32.3 \%)$, retired 
$(n=45 ; 72.6 \%)$, income up to one salary $(n=44 ; 71.0 \%)$ and incomplete elementary school $(n=35 ; 56.5 \%)$. Satisfaction was significantly higher in CD users in all parameters evaluated $(\mathrm{p}<0.05)$. Among the indicators of quality of life, only physical pain $(\mathrm{p}=0.123)$ and psychological limitation $(\mathrm{p}=0.087)$ showed no statistically significant difference ( $>0.05)$. Conclusion: Within the limitations of this study, rehabilitation with CD promoted a positive impact on the life of the elderly, guaranteeing greater satisfaction and better quality of life. In addition, the VAS and adjective scales presented substantial agreement.

Keywords: Complete denture; Patient satisfaction; Quality of life.

\begin{abstract}
Resumen
Objetivo: Comparar la satisfacción y la calidad de vida de los usuarios de edad avanzada y los no usuarios de prótesis completas (pero que requieren prótesis). Métodos: se trata de un estudio transversal. La muestra estuvo formada por 62 ancianos, 31 usuarios (G1) y 31 no usuarios (G2) de prótesis total (TP). Para todos los participantes, se aplicaron cuestionarios de satisfacción (Gurgel et al., 2015) y calidad de vida (Perfil de Impacto en la Salud Oral para desdentados - OHIP-Edent). Los datos se analizaron mediante análisis descriptivo, seguido de pruebas estadísticas para evaluar las diferencias en satisfacción y calidad de vida entre usuarios y no usuarios de TP. Resultados: El análisis comparativo de los aspectos sociodemográficos entre G1 y G2 no mostró diferencia estadísticamente significativa $(p>0.05)$. La mayoría eran mujeres $(n=51 ; 82,3 \%)$, casadas $(n=20 ; 32,3 \%)$, jubiladas $(n=45 ; 72,6 \%)$, con ingresos de hasta 1 salario $(n=44 ; 71,0 \%)$ y educación primaria incompleta $(n=35 ; 56,5 \%)$. La satisfacción fue significativamente mayor entre los usuarios de TP en todos los parámetros evaluados $(\mathrm{p}<0,05)$. Entre los indicadores de calidad de vida, solo el dolor físico $(\mathrm{p}=0,123)$ y la limitación psicológica $(\mathrm{p}=0,087)$ no mostraron diferencia estadísticamente significativa ( $p>0,05$ ). Conclusión: Dentro de las limitaciones de este estudio, la rehabilitación con TP promovió un impacto positivo en la vida de las personas mayores, al asegurar una mayor satisfacción y una mejor calidad de vida. Además, la EVA y las escalas de adjetivos mostraron un acuerdo sustancial.
\end{abstract}

Palabras clave: Prótesis dental total; Satisfacción del paciente; Calidad de vida.

\title{
1. Introdução
}

O envelhecimento populacional ativo e revolução demográfica, caracterizada pelo aumento na proporção de idosos, constitui um desafio para os gestores públicos na perspectiva de ser ofertada uma atenção adequada às suas necessidades (Kindig, Nobles, \& Zidan 2018). O aumento significativo na população de idosos não está sendo acompanhada de modificações suficientes no atendimento às suas necessidades de saúde bucal. Assim, torna-se imprescindível o aumento da oferta, capacitação dos profissionais e melhoria do sistema de referência, visando integralidade da atenção à saúde e aumento da capacidade de resolução do sistema (Nobrega et al., 2016, Silva, Demarco, \& Feldens, 2015).

No campo da saúde bucal, a população idosa carrega a herança da prática assistencialista, que resultou em elevada prevalência de edentulismo e acúmulo da necessidade de prótese dentária. De acordo com a Organização Mundial de Saúde (OMS), o edentulismo é resultado de um sistema de saúde deficiente e afeta consideravelmente o padrão de saúde bucal e geral das populações (Cardoso, Balducci, Telles, Lourenço, \& Nogueira Júnior, 2016; Bortoli, Moreira, Moretti-Pires, Botazzo, \& Kovaleski, 2017). Dados do SB Brasil 2010 demonstraram que houve melhoria nas condições de saúde bucal da população adulta quanto à demanda por próteses dentárias. Contudo, o grande problema ainda se concentra na população entre 65 a 74 anos, que apresentou CPO-D médio de 27,1 em 2010 (em 2003 a média era de 27,8) com predominância do componente perdido por cárie (Brasil, 2012).

Sabe-se, portanto, que as iniquidades sociais interferem não somente na saúde bucal, como também na saúde geral das populações, fazendo com que indivíduos que residem em áreas com grandes diferenças de renda apresentem piores condições bucais em relação àqueles com situação socioeconômica semelhante, mas que vivem em regiões com menor disparidade econômica (Lamy, Andrade, \& Matta, 2020). Seja qual for o tipo de prótese dentária utilizada, a reabilitação oral influencia psicossocialmente a vida do paciente. A ausência de elementos dentários não é uma consequência normal do envelhecimento, mas o resultado de eventos ao longo da vida, que interferem diretamente em atividades sociais, reduz a autoconfiança, causa um envelhecimento prematuro, altera a autoimagem, reduz a socialização e aumenta o risco na má nutrição (Rodrigues et al., 2015, Gil-Montoya, Mello, Barrios, Gonzalez-Moles, \& Bravo, 2015). Assim, mais do que restabelecer a função mastigatória 
ao procurar o tratamento reabilitador, os pacientes buscam reconstituir sua imagem pessoal, social e sua qualidade de vida (Kreve, D’Avila, Santos, \& Reis, 2020).

Muitas pesquisas fazem uso de questionários validados na literatura e os resultados que estes instrumentos geram são fundamentais, pois trazem informações relevantes sobre um determinado grupo. Dentre estes questionários, dá-se ênfase àqueles usados para avaliar a satisfação e qualidade de vida (Oral Health Impact Profile for edentulous - OHIP-Edent), pois eles são fundamentais e podem ser usados para estimar a satisfação e qualidade de vida das pessoas, bem como dar subsídios à instituição de medidas educativas. Assim, o objetivo deste estudo foi comparar a satisfação e qualidade de vida de idosos usuários e não usuários de prótese total (PT), por meio de instrumentos já consolidados na literatura. Para tanto, a hipótese nula de que não há diferença na satisfação e qualidade de vida entre usuários e não usuários de PT foi testada.

\section{Metodologia}

Trata-se de uma pesquisa de campo quantitativa que teve a finalidade de observar fatos e fenômenos em uma população específica de idosos e, por meio da aplicação de questionários, foi possível mensurar e quantificar as respostas dos entrevistados para obtenção de dados que poderiam confirmar ou contestar as hipóteses iniciais do estudo (Estrela, 2018). Assim, este trabalho descreve os resultados de um estudo transversal, cuja informação foi coletada por meio de questionários de satisfação e qualidade de vida aplicados a dois grupos distintos de idosos: usuários de prótese total (G1) e não usuários mas que necessitam de prótese (G2). A pesquisa foi aprovada pelo Comitê de Ética em Pesquisa da Universidade de Pernambuco (CEP-UPE) sob parecer $n^{\circ}$ 2.576.315 e registro CAAE 85376018.0.0000.5207.

\subsection{Participantes}

O estudo foi realizado no Centro de Atenção ao Idoso (CAI), localizado no município de Arcoverde, no estado de Pernambuco. Os critérios de inclusão foram os seguintes: (1) idosos usuários de prótese total (superior e inferior) ou não usuários (mas que necessitam de prótese) que frequentaram o CAI durante a coleta dos dados, realizada entre meses de abril a junho de 2018; (2) idade de 60 anos ou mais, de ambos os sexos; (4) boa compreensão do português falado; (4) boa receptividade e (5) assinado o TCLE. Foram excluídos aqueles que tinham déficit cognitivo que os impediam de compreender o objetivo do estudo.

\subsection{Cálculo do tamanho da amostra}

Inicialmente, foi realizado um estudo piloto com 20 indivíduos, sendo 10 de cada grupo. O cálculo amostral foi realizado com base na diferença das médias entre os grupos do estudo da variável "qualidade de vida (OHIP-Edent)", utilizando o software estatístico OpenEpi 3.01 Web (Dean, Sullivan, \& Soe, 2013). Os parâmetros usados para este cálculo foram: intervalo de confiança (95\%), poder do teste (95\%), diferença de médias $(6,7)$ e desvio padrão (Não usuários: 7.5 / Usuários: 6.9) dos grupos. Com bases nestes parâmetros, um tamanho amostral de 62 participantes (31 para cada grupo) foi suficiente para detectar diferenças relevantes $(\alpha=0,05 ; \beta=0,05)$.

\subsection{Coleta dos dados}

Foram colhidos dados referentes à idade, gênero, renda, estado civil, atividade profissional e escolaridade. Na sequência, os participantes responderam os questionários referentes à satisfação e qualidade de vida.

A variável satisfação foi avaliada com o mesmo questionário utilizado por Gurgel et al. (2015), que contém 5 itens e versa questões sobre a satisfação geral, mastigação, estética, conforto e fala. Para cada questão, a satisfação foi quantificada 
por meio da Escala visual analógica (EVA) medindo $100 \mathrm{~mm}$, ancorada pelas palavras "nem um pouco satisfeito" e "extremamente satisfeito", desenvolvida por Awad e Feine (1998). Os participantes foram instruídos a marcarem uma linha perpendicular à reta da EVA no ponto que corresponde ao seu nível de satisfação. O valor é definido, portanto, como a distância em milímetros da extremidade esquerda da reta até a marcação realizada pelo participante.

A variável qualidade de vida foi avaliada através do OHIP-Edent, conforme validação da versão brasileira por Souza, Patrocínio, Pero, Marra, e Compagnoni (2007). Trata-se de um instrumento subjetivo que julga a percepção do indivíduo em relação à sua saúde bucal, levando em consideração a expectativa na qualidade de vida. É composto por 19 questões, divididas em sete domínios, que procura investigar: (1) Limitação funcional; (2) Dor física; (3) Desconforto psicológico; (4) Limitação física; (5) Limitação Psicológica; (6) Limitação social e (7) Incapacidade devido a problemas com dentes, boca ou dentadura. Para cada pergunta, foi dada uma das seguintes respostas: (0) Nunca; (1) Às vezes e (2) Quase sempre. A somatória das pontuações de todas as perguntas gerou o índice OHIP-Edent, variando entre 0 a 38. Quanto maior o índice OHIP-Edent, maior a percepção de impacto negativo da saúde bucal na qualidade de vida do indivíduo.

\subsection{Análise estatística}

Os dados foram tabulados no Statistical Package for the Social Sciences - SPSS ${ }^{\circledR} 17.0$ (SPSS Inc., Chicago, USA) e analisados inicialmente por meio de estatística descritiva. Diferenças estatísticas foram avaliadas entre os grupos (Usuários e não usuários) e variáveis sociodemográficas, tais como idade (t de Student), gênero (Exato de Fisher), estado civil, atividade profissional, escolaridade e renda (Qui-quadrado). As questões relacionadas à satisfação e qualidade de vida de usuários e não usuários de prótese total foram avaliadas por meio do Teste t de Student. Para todas as análises, o nível de significância usado foi de $5 \%$.

\section{Resultados}

A Figura 1 apresenta o diagrama de fluxo dos participantes. Inicialmente, 88 participantes que frequentarem o CAI nos meses de abril a junho de 2018 foram avaliados para elegibilidade, dos quais 52 eram usuários de PT (G1) e 36 não usuários (G2). Desse total, 26 participares foram excluídos, com base nos critérios de inclusão e exclusão, sendo 21 usuários de PT (G1) e 5 não usuários (G2).

Figura 1. Diagrama de fluxo dos participantes.

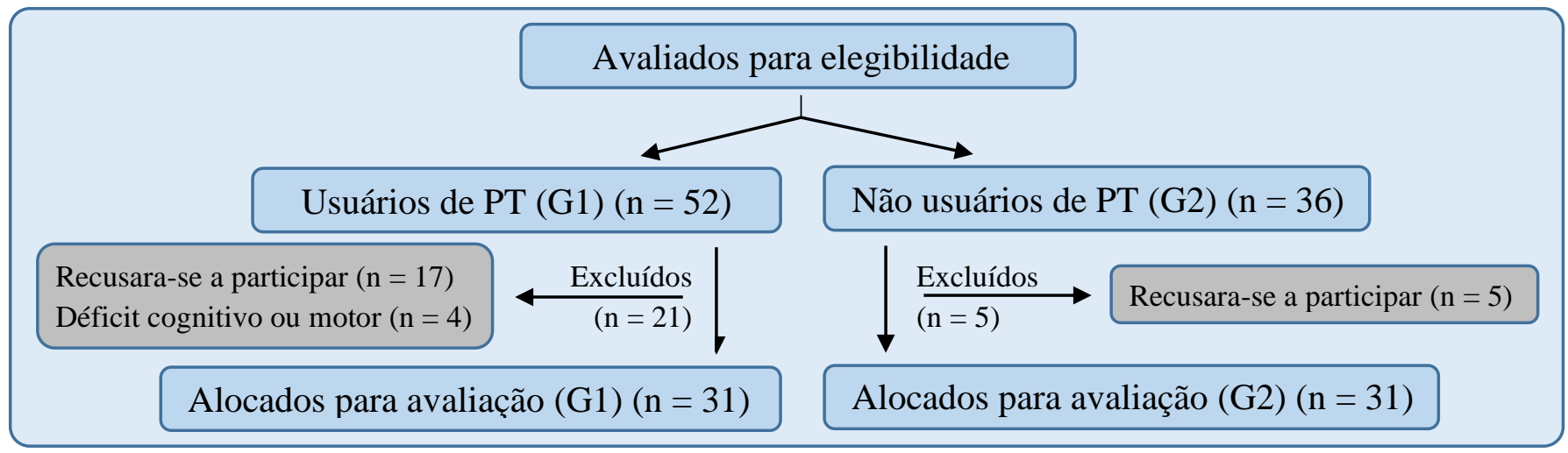

Fonte: Autores (2020).

Um total de 62 idosos usuários de PT participou deste estudo, dos quais 31 são usuários de PT (G1) e 31 não usuários de PT, mas que necessitam de prótese (G2). A média de idade foi de 69,1 7 ,1, variando entre 61 e 89 . A maioria foi do gênero 
feminino $(n=51 ; 82,3 \%)$, casado $(n=20 ; 32,3 \%)$, aposentado $(n=45 ; 72,6 \%)$, renda de até 1 salário $(n=44 ; 71,0 \%)$ e ensino fundamental incompleto $(\mathrm{n}=35 ; 56,5 \%)$. A análise comparativa dos aspectos sociodemográficos (idade, gênero, estado civil, atividade profissional, renda e escolaridade) entre usuários e não usuários de PT mostrou nenhuma diferença estatisticamente significante ( $\mathrm{p}>0,05)$, mostrando que se trata de uma amostra homogênea (Tabela 1).

Tabela 1. Caracterização sóciodemográfica da amostra.

\begin{tabular}{|c|c|c|c|c|}
\hline \multirow{2}{*}{ Característica } & \multicolumn{2}{|c|}{ Grupo } & \multirow{2}{*}{ Total } & \multirow{2}{*}{$\mathbf{p}$} \\
\hline & Usuário & Não usuário & & \\
\hline Idade $($ anos) $*$ & $70,7 \pm 8,0$ & $67,0 \pm 5,2$ & $69,1 \pm 7,1$ & $0,530^{\dagger}$ \\
\hline \multicolumn{5}{|l|}{ Gênero** } \\
\hline Masculino & $4(6,5)$ & $7(11,3)$ & $11(17,7)$ & $0,508^{\ddagger}$ \\
\hline Feminino & $27(43,5)$ & $24(38,7)$ & $51(82,3)$ & \\
\hline Total & $31(50,0)$ & $31(50,0)$ & $62(100,0)$ & \\
\hline \multicolumn{5}{|l|}{ Estado civil** } \\
\hline Solteiro/Divorciado & $9(14,5)$ & $15(24,2)$ & $24(38,7)$ & $0,085^{\#}$ \\
\hline Casado & $14(22,6)$ & $6(9,7)$ & $20(32,3)$ & \\
\hline Viúvo & $8(12,9)$ & $10(16,1)$ & $18(29,0)$ & \\
\hline Total & $31(50,0)$ & $31(50,0)$ & $62(100,0)$ & \\
\hline \multicolumn{5}{|l|}{ Atividade profissional** } \\
\hline Aposentado & $25(40,3)$ & $20(32,3)$ & $45(72,6)$ & $0,326^{\#}$ \\
\hline Dono(a) de casa & $2(3,2)$ & $5(8,1)$ & $7(11,3)$ & \\
\hline Empregado/Autônomo & $4(6,5)$ & $6(9,7)$ & $10(16,1)$ & \\
\hline Total & $31(50,0)$ & $31(50,0)$ & $62(100,0)$ & \\
\hline \multicolumn{5}{|l|}{ Escolaridade** } \\
\hline Não alfabetizado & $5(8,1)$ & $4(6,5)$ & $9(14,5)$ & $0,087^{\#}$ \\
\hline Ens. fund. incompleto & $18(29,0)$ & $17(27,4)$ & $35(56,5)$ & \\
\hline Ens. fund. completo & $0(0,0)$ & $6(9,7)$ & $6(9,7)$ & \\
\hline Ens. médio completo & $6(9,7)$ & $2(3,2)$ & $8(12,9)$ & \\
\hline Ens. superior completo & $2(3,2)$ & $2(3,2)$ & $4(6,5)$ & \\
\hline Total & $31(50,0)$ & $31(50,0)$ & $62(100,0)$ & \\
\hline \multicolumn{5}{|l|}{ Renda** } \\
\hline Até 1 salário & $19(30,6)$ & $25(40,3)$ & $44(71,0)$ & $0,110^{\#}$ \\
\hline Entre 1 e 2 salários & $9(14,5)$ & $6(9,7)$ & $15(24,2)$ & \\
\hline Mais de 2 salários & $3(4,8)$ & $0(0,0)$ & $3(4,8)$ & \\
\hline Total & $31(50,0)$ & $31(50,0)$ & $62(100,0)$ & \\
\hline
\end{tabular}

Dados comparados por meio do Teste t de Student ${ }^{\dagger}$, Exato de Fisher ${ }^{\ddagger}$ e Qui-quadrado ${ }^{\#}$.

* Média \pm desvio padrão; **n $(\%)$.

Fonte: Autores (2020).

A satisfação foi significativamente maior nos usuários de PT em todos os parâmetros avaliados, incluindo mastigação

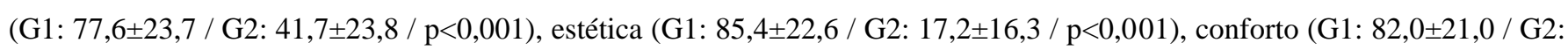




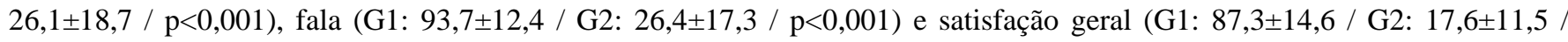
$\mathrm{p}<0,001)$ (Tabela 2).

Tabela 2. Frequência absoluta (n), média (desvio padrão, SD) e diferença entre intervalos de confiança para a variável satisfação, em função dos grupos (usuários e não usuários de prótese total).

\begin{tabular}{|c|c|c|c|c|c|}
\hline Satisfação & $\mathbf{n}$ & Média (SD) & $\begin{array}{c}\text { Diferença média entre grupos } \\
\text { (IC95) }\end{array}$ & $\mathbf{t}^{\dagger}$ & $\mathbf{p}$ \\
\hline \multicolumn{6}{|l|}{ Mastigação } \\
\hline Usuário & 31 & $77,6(23,7)$ & $35,9(22,9-48,9)$ & 5,522 & $<0,001 *$ \\
\hline Não Usuário & 31 & $41,7(23,8)$ & & & \\
\hline \multicolumn{6}{|l|}{ Estética } \\
\hline Usuário & 31 & $85,4(22,6)$ & $68,3(57,2-79,3)$ & 12,417 & $<0,001 *$ \\
\hline Não Usuário & 31 & $17,2(16,3)$ & & & \\
\hline \multicolumn{6}{|l|}{ Conforto } \\
\hline Usuário & 31 & $82,0(21,0)$ & $55,9(44,9-66,9)$ & 10,197 & $<0,001 *$ \\
\hline Não Usuário & 31 & $26,1(18,7)$ & & & \\
\hline \multicolumn{6}{|l|}{ Fala } \\
\hline Usuário & 31 & $93,7(12,4)$ & $67,3(59,2-75,4)$ & 16,655 & $<0,001 *$ \\
\hline Não Usuário & 31 & $26,4(17,3)$ & & & \\
\hline \multicolumn{6}{|l|}{ Geral } \\
\hline Usuário & 31 & $87,3(14,6)$ & $69,8(62,4-77,1)$ & 19,162 & $<0,001 *$ \\
\hline Não Usuário & 31 & $17,6(11,5)$ & & & \\
\hline
\end{tabular}

$\dagger$ Teste $\mathrm{t}$ de Student.

* Diferença estatisticamente significante $(\mathrm{p}<0,05)$.

Fonte: Autores (2020).

Em relação à qualidade de vida, usuários de PT mostraram ter melhor qualidade vida nos seguintes indicadores:

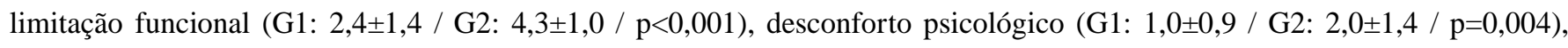

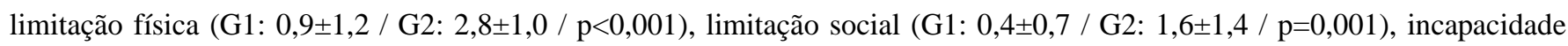

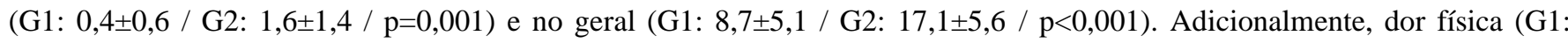

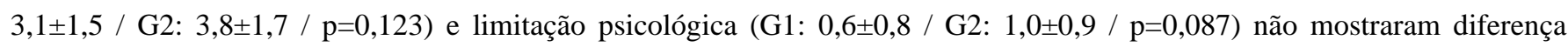
estatisticamente significante (Tabela 3). 
Tabela 3. Frequência absoluta (n), média (desvio padrão), mínimo (mín.), primeiro quartil (Q25), mediana, segundo quartil (Q75) e máximo para a variável qualidade de vida, em função dos grupos (usuários e não usuários de prótese total).

\begin{tabular}{|c|c|c|c|c|c|c|c|c|c|}
\hline OHIP-Edent ${ }^{\#}$ & $\mathbf{n}$ & Média (SD) & Mín. & Q25 & Mediana & Q75 & Máx. & $\mathbf{t}^{\dagger}$ & $\mathbf{p}$ \\
\hline \multicolumn{10}{|l|}{ Limitação Funcional } \\
\hline Não usuário & 31 & $4,3(1,0)$ & 2 & 4 & 5 & 5 & 6 & 5,481 & $<0,001 *$ \\
\hline Usuário & 31 & $2,4(1,4)$ & 0 & 1 & 2 & 4 & 5 & & \\
\hline \multicolumn{10}{|l|}{ Dor física } \\
\hline Não usuário & 31 & $3,8(1,7)$ & 1 & 2 & 3 & 5 & 7 & 1,568 & 0,123 \\
\hline Usuário & 31 & $3,1(1,5)$ & 0 & 2 & 3 & 4 & 6 & & \\
\hline \multicolumn{10}{|c|}{ Desconforto psicológico } \\
\hline Não usuário & 31 & $2,0(1,4)$ & 0 & 1 & 2 & 3 & 4 & 3,049 & $0,004^{*}$ \\
\hline Usuário & 31 & $1,0(0,9)$ & 0 & 0 & 1 & 2 & 3 & & \\
\hline \multicolumn{10}{|l|}{ Limitação física } \\
\hline Não usuário & 31 & $2,8(1,0)$ & 2 & 2 & 2 & 3 & 5 & 6,121 & $<0,001 *$ \\
\hline Usuário & 31 & $0,9(1,2)$ & 0 & 0 & 0 & 1 & 5 & & \\
\hline \multicolumn{10}{|l|}{ Limitação psicológica } \\
\hline Não usuário & 31 & $1,0(0,9)$ & 0 & 0 & 1 & 2 & 3 & 1,744 & 0,087 \\
\hline Usuário & 31 & $0,6(0,8)$ & 0 & 0 & 0 & 1 & 2 & & \\
\hline \multicolumn{10}{|l|}{ Limitação social } \\
\hline Não usuário & 31 & $1,6(1,4)$ & 0 & 0 & 1 & 3 & 4 & 3,729 & $0,001 *$ \\
\hline Usuário & 31 & $0,4(0,7)$ & 0 & 0 & 0 & 1 & 3 & & \\
\hline \multicolumn{10}{|l|}{ Incapacidade } \\
\hline Não usuário & 31 & $1,6(1,4)$ & 0 & 0 & 1 & 3 & 4 & 3,712 & $0,001 *$ \\
\hline Usuário & 31 & $0,4(0,6)$ & 0 & 0 & 0 & 1 & 2 & & \\
\hline \multicolumn{10}{|l|}{ Geral } \\
\hline Não usuário & 31 & $17,1(5,6)$ & 8 & 14 & 17 & 21 & 27 & 5,468 & $<0,001 *$ \\
\hline Usuário & 31 & $8,7(5,1)$ & $\mathbf{0}$ & 5 & 7 & 12 & 20 & & \\
\hline
\end{tabular}

\# Do inglês "Oral Health Profile for assessing edentulous subjects" para avaliar qualidade de vida relacionada à saúde bucal de pacientes edêntulos.

$\dagger$ Teste $t$ de Student.

* Diferença estatisticamente significante $(\mathrm{p}<0,05)$.

Fonte: Autores (2020).

\section{Discussão}

A saúde bucal constitui parte integrante da saúde geral e contribui para a qualidade de vida do indivíduo. É necessário, portanto, uma extensa compreensão do impacto da reabilitação oral para que os profissionais se tornem capazes de definir os benefícios causados por esta modalidade de tratamento (Beloni, Vale, \& Takahashi, 2013; Kreve et al., 2020). Segundo a Pesquisa Nacional de Saúde Bucal realizada pelo SUS em 2010 apontou que, dentre os idosos examinados, 23\% necessitavam de prótese total em pelo menos um dos arcos, e 15\% necessitavam de próteses totais bimaxilares. Ainda nesse estudo projetouse que a taxa de edentulismo para idosos tende a aumentar até 2040, podendo atingir cerca de 64 milhões de mandíbulas desdentadas, podendo assim, afirmar que o assunto é de suma importância para o bem-estar de uma grande parcela da população (Cardoso et al., 2016) 
A população do presente estudo foi composta, em sua grande maioria, por idosos com renda familiar em torno de 954 reais, corroborando com Menezes, Bertolini e Massuda (2020). Houve maior frequência de mulheres, uma vez que elas procuram os serviços de saúde pública com mais frequência, seja pela maior proatividade, por participarem em atividades em grupo e estão com mais frequência em casas de apoio e instituições de longa permanência, locais em que são realizados estudos de mesmo escopo (Gabardo et al., 2015, Soares, Rodrigues, Ribeiro, \& Rosendo, 2015).

Grande parte dos estudos na literatura que avaliaram pacientes reabilitados com PT mostraram resultados consideravelmente altos de satisfação e qualidade de vida. De forma semelhante, os resultados deste estudo revelaram satisfação e qualidade de vida consideravelmente superiores em relação àquele grupo de idosos não usuários de PT em quase todos os parâmetros avaliados (Tabelas 2 e 3), negando, portanto a primeira hipótese nula do estudo. Nessa perspectiva, estes dados podem fornecer ao profissional a noção do impacto que a reabilitação oral pode causar na qualidade de vida dos indivíduos. Apesar das deficiências funcionais das PTs, a maioria dos pacientes encontrava-se satisfeita $(87,3 \pm 14,6) \mathrm{em}$ consonância com outros estudos que mostram a variação média de satisfação em torno de 50\% a 75\% (Turker, Sener, \& Ozkan, 2009)

As razões mais comuns de insatisfação em usuários de PT observadas neste estudo foram a estética, seguida do conforto/fala e, por último a mastigação. Estes achados sugerem que os idosos são mais preocupados com a estética e a implicação social da reabilitação do que propriamente a função que elas desenvolvem no sistema estomatognático. Albaker (2013) e Kreve et al. (2020) observaram que a incapacidade funcional e social, a dor e o desconforto psicológico foram situações mais frequentemente relatadas por pacientes portadores de prótese total bimaxilar. Assim, o envelhecimento por si só é um fator que interfere no aumento da probabilidade de desenvolvimento de distúrbios de voz, pois fatore anatômicos e funcionais como cartilagem e os músculos que participam da fonação também sofrem alterações estruturais, na intensidade e na qualidade vocal (Roy, Kim, Courey, \& Cohen, 2016).

O envelhecimento também causa prejuízo nos hábitos alimentares, tornando os alimentos fundamentais menos atraentes e levando a deficiências nutricionais. Esses fatores podem gerar limitações no sistema imunológico, aumentar o risco de infecções e fraqueza muscular (Amarya, Singh, \& Sabharwal, 2015). O uso de próteses em idosos aumenta o desconforto durante a mastigação, tornando assim a dieta pobre em nutrientes (Silva et al., 2015; Menezes et al., 2020). Assim, a reabilitação oral de idosos com próteses totais, associada a aconselhamento dietético aumenta a ingestão de alimentos saudáveis e melhora a qualidade de vida (Amagai et al., 2017), além de afetar positivamente a qualidade de vida (Kreve et al., 2020).

O OHIP foi desenvolvido para avaliar o impacto social (bem-estar e qualidade de vida) causado pela condição de saúde bucal nos quesitos limitação funcional, dor física, desconforto psicológico, incapacidade física, psicológica e social e deficiência na realização das atividades cotidianas (Miotto, Barcelos, \& Velten, 2012). De acordo com os resultados do OHIPEdent, os idosos reabilitados com PT $(8,7 \pm 5,1)$ relataram melhor índice de qualidade de vida em comparação aos idosos não usuários de PT $(17,1 \pm 5,6)$, indicando maior aceitação e adaptação às suas reabilitações. Esses achados corroboram com o estudo realizado por Beloni et al. (2013). Todavia, considerando que a pior qualidade de vida teria uma média de 38,0, os não usuários de PT não atingiram nem 50\%, sugerindo que a qualidade de vida dos idosos não está tão comprometida, especialmente nos parâmetros limitação psicológica e dor física que não apresentaram diferença estatisticamente significante $(\mathrm{p}>0,05)$.

Inúmeros trabalhos científicos avaliam o resultado de tratamentos odontológicos por meio de parâmetros objetivos, desconsiderando, portanto, a relevância dos achados subjetivos. Entretanto, alguns estudiosos sugerem que os indicadores subjetivos, tais como satisfação e qualidade de vida, devem ser usados na mensuração da percepção da saúde bucal, complementando, dessa forma, informações da necessidade de tratamento reabilitador protético (Pocztaruk et al., 2006). Além 
disso, esses indicadores podem ser usados para complementar informações clínicas dos pacientes idosos, revelando sua expectativa com a própria saúde em vários aspectos e tendo o propósito maior de avaliar a sua qualidade de vida (Vargas \& Paixão, 2005).

Apesar dos resultados impactantes observados no estudo, o ideal seria avaliar o paciente longitudinalmente, antes e após a reabilitação protética. Somente desta forma, seria possível afirmar, de fato, o real impacto da reabilitação na satisfação e qualidade de vida dos idosos, pois quando inclui-se diferentes participantes em dois grupos para verificar o efeito de uma reabilitação, não estamos levando em conta a subjetividade de cada um, embora diferença estatisticamente significante não tenha sido verificada nos aspectos sociodemográficos entre os dois grupos. Assim, sugere-se novos estudos com grupos pareados para evitar vieses de subjetividade dos participantes.

\section{Conclusão}

Dentro das limitações deste estudo, a reabilitação com PT promoveu um impacto positivo na vida dos idosos, ao garantir uma maior satisfação e melhor qualidade de vida.

\section{Referências}

Albaker, A. M. (2013). The oral health-related quality of life in edentulous patients treated with conventional complete dentures. Gerodontology, 30(1), 61-6. 10.1111/j.1741-2358.2012.00645.x.

Amagai, N., Komagamine, Y., Kanazawa, M., Iwaki, M., Jo, A., Suzuki, H., \& Minakuchi, S. (2017). The effect of prosthetic rehabilitation and simple dietary counseling on food intake and oral health related quality of life among the edentulous individuals: a randomized controlled trial. $J$ Dent, 65 , 89-94. 10.1016/j.jdent.2017.07.011.

Amarya, S., Singh, K., \& Sabharwal, M. (2015). Changes during aging and their association with malnutrition. J Clin Gerontol Geriatr, 6(3), 78-84. 10.1016/j.jpgg.2015.05.003

Awad, M. A., \& Feine, J. S. (1998). Measuring patient satisfaction with mandibular prostheses. Community Dent Oral Epidemiol, 26(6), 400-405. 10.1111/j.1600-0528.1998.tb01978.x.

Beloni, W. B., Vale, H. F., \& Takahashi, J. M. F. K. (2013). Assessment of the satisfaction level and quality of life of dental prosthesis users. Rev Facul Odontol, 18(2), 160-164. 10.5335/rfo.v18i2.3255.

Bortoli, F. R., Moreira, M. A., Moretti-Pires, R. O., Botazzo, C., \& Kovaleski, D. F. (2017). Percepção da saúde bucal em mulheres com perdas dentárias extensas. Saúde Soc, 26(2), 533-544. 10.1590/s0104-12902017162160.

Brasil, Ministério da Saúde. (2012). Secretaria de Atenção à Saúde. Departamento de Atenção Básica. Projeto SB Brasil 2010: Resultados principais. Brasília, DF.

Cardoso, M., Balducci, I., Telles, D. M., Lourenço, E.J., \& Nogueira Júnior, L. (2016). Edentulism in Brazil: trends, projections and expectations until 2040. Cien Saude Colet, 21(4), 1239-1246. 10.1590/1413-81232015214.13672015.

Dean, A. G., Sullivan, K. M., \& Soe, M. M. (2013). OpenEpi: Open Source Epidemiologic Statistics for Public Health, Versão 3.01. http://www.openepi.com/ SampleSize/SSMean.htm.

Estrela, C. (2018). Metodologia científica: Ciência, ensino, pesquisa. Artes Médicas.

Gabardo, M. C. L., Moysés, S. J., Moysés, S. T., Olandoski, M., Olinto, M. T. A., Pattussi, \& M. P. (2015). Multilevel analysis of self-perception in oral health and associated factors in Southern Brazilian adults: a cross-sectional study. Cad. Saúde Pública, 31(1), 49-59. 10.1590/0102-311X00037814.

Gil-Montoya, J. A., Mello, A. L., Barrios, R., Gonzalez-Moles, M. A., \& Bravo, M. (2015). Oral health in the elderly patient and its impact on generalwellbeing: a nonsystematic review. Clin Interv Aging, 10, 461-467. 10.2147/CIA.S54630.

Gurgel, B. C. V., Pascoal, A. L. B., Souza, B. L. M., Dantas, P. M. C., Montenegro, S. C. L., Oliveira, A. G. R. C., \& Calderon, P. S. (2015). Patient satisfaction concerning implant-supported prostheses: an observational study. Braz Oral Res, 29(1): 1-6. 10.1590/1807-3107BOR-2015.vol29.0034.

Kindig, D., Nobles, J., \& Zidan, M. (2018). Meeting the Institute of Medicine's 2030 US Life Expectancy Targe. Am J Public Health, 108(1), 87-92. 10.2105/AJPH.2017.304099.

Kreve, S., D’Avila, G. C., Santos, L. O., \& Reis, A. C. (2020). Autopercepção da saúde bucal de idosos. Clin Lab Res Den, 2020, 1-9. http://dx.doi.org/10.11606/issn.2357-8041.clrd.2020.160816.

Lamy, R. D. L. R. F., Andrade, C. L. T., \& Matta, G. C. (2020). Iniquidades sociais e saúde bucal: revisão integrativa. Rev Aten Saúde, 18(63), 82-98. 10.13037/ras.vol18n63.6094. 
Research, Society and Development, v. 10, n. 4, e56010414614, 2021

(CC BY 4.0) | ISSN 2525-3409 | DOI: http://dx.doi.org/10.33448/rsd-v10i4.14614

Menezes, T., Bertolini, S. M. M. G., \& Massuda, E. M. (2020). Autopercepção da saúde bucal a usuários de prótese, adultos mais velhos. Rev. Kairós, 23(1), 47-63. http://dx.doi.org/10.23925/2176-901X.20209v23i1p47-63.

Miotto, M. H. M. B, Barcelos, L. A., \& Velten, D. B. (2012). Avaliação do impacto na qualidade de vida causado por problemas bucais na população adulta e idosa em município da Região Sudeste. Ciênc Saúde Colet, 17(2), 397-406. 10.1590/S1413-81232012000200014.

Nobrega, D. R. M, Lucena, A. G., Medeiros, L. A. D. M., Farias, T. S. S., Meira, K. R. S., \& Mahon, S. M. O. D. (2016). Avaliação da utilização e hábitos de higiene em usuários de prótese dentária removível. Rev Bras Odontol, 73(3), 193-197. http://dx.doi.org/10.18363/rbo.v73n3.p.193.

Pocztaruk, R. L., Frasca, L. C. F., Rivaldo, E. G., Mattia, P. R. C., Vidal, R. A., Fernandes, E., \& Gavião, M. B. D. (2006). Satisfaction level and masticatory capacity in edentulous patients with conventional dentures and implant-retained overdentures. Brazilian Journal of Oral Sciences, 5(19), 1232-1238. https://doi.org/10.20396/bjos.v5i19.8642953.

Rodrigues, J. H., Marques, M. M., Biasotto-Gonzalez, D. A., Moreira, M. S., Bussadori, S. K., Mesquita-Ferrari, R. A., \& Martins, M. D. (2015). Evaluation of pain, jaw movements, and psychosocial factors in elderly individuals with temporomandibular disorder under laser phototherapy. Lasers Med Sci, 30(3), 953-959. 10.1007/s10103-013-1514-z.

Roy, N., Kim, J., Courey, M., \& Cohen, S. M. (2016). Voice disorders in the elderly: a national database study. Laryngoscope, 126(2), 421-428. 10.1002/lary.25511.

Silva, M. A., Batista, A. U. D., Abreu, M. H. N. G., \& Forte, F. D. S. (2020). Impact on the Quality of Life of Older Adults Who Use Inadequate Dental Prostheses: A Cross-Sectional Study. Pesqui. Bras. Odontopediatria Clín. Integr, 20, 4713. https://doi.org/10.1590/pboci.2020.074.

Soares, S. L. B., Rodrigues, R. A., Ribeiro, R. A., \& Rosendo, R. A. (2015). Avaliação dos usuários de prótese total, abordando aspectos funcionais, sociais e psicológicos. Rev Saúde Ciênc, 4(2), 19-25.

Souza, R. F., Patrocínio, L., Pero, A. C., Marra, J., \& Compagnoni, M. A. (2007). Reliability and validation of a Brazilian version of the Oral Health Impact Profile for assessing edentulous subjects. J Oral Rehabil, 34(11), 821-826. 10.1111/j.1365-2842.2007.01749.x.

Turker, S. B., Sener, I. D., \& Ozkan, Y. K. (2009). Satisfaction of the complete denture wearers related to various factors. Arch Gerontol Geriatr, 49(2), 126129. 10.1016/j.archger.2008.11.003.

Vargas, A. M. D., \& Paixão, H. H. (2005). Perda dentária e seu significado na qualidade de via de adultos usuários de serviço público de saúde bucal do Centro de Boa Vista, em Belo Horizonte. Cien Saúde Colet, 10(4), 1015-1024. http://dx.doi.org/10.1590/S1413-812320050004 